\title{
XXVII.
}

Arbeiten aus dem Laboratorium für experimentelle Pharmakologie zu Straßburg.

\section{8. Über das Verhalten des Akridins im Organismus des} Kaninchens.

Von

Dr. phil. et med. Hermann Fühnex.

Im Jahre 1887 machte W. $\mathrm{H}$ is ${ }^{1}$ ) im Pharmakologischen Institute zu Straßburg die wichtige Beobachtung, daß Pyridin, an Hunde verfuttert, im Urin derselben als Methylpyridiniumhydroxyd (Methylpyridinammoniumhydroxyd) ausgeschieden wird.

Die Homologen des Pyridins hingegen, die Methylpyridine, werden nach den Untersuchungen von $\mathrm{R}$. Cohn ${ }^{2}$ ) nicht am Stickstoff methyliert, sondern in der Seitenkette, der Methylgruppe, oxydiert und treten als Pyridincarbonsäuren im Harn auf.

In welcher Form in den Magen eingeftihrtes Chinolin den Tierkörper verläßt, ist heute noch nicht sicher bekannt, trotz verschiedener Untersuchungen ${ }^{3}{ }^{4}$ ), die in den achtziger Jahren des vorigen Jahrhunderts angestellt wurden, als man versuehte, das Chinolin in der Fiebertherapie zu verwerten. Ob auch hier eine Methylierung am Stickstoff stattfindet, wie beim Pyridin, ob der Benzolkern zu Phenol oxydiert wird, ob der Benzolkern beziehungsweise der Pyridinkern aufgespalten wird und sich Pyridincarbonsäuren, Benzoesäure n. a. im Harn fiuden, sind noch offene Fragen.

Ich beabsichtigte daher, die Stoffwechselprodukte des Chinolins von neuem zu untersuchen. Vorher aber wollte ich das Verhalten

1) W. His, Ũber das Stoffwechselprodukt des Pyridins. Dieses Archiv. 22 (1887). S. 253.

2) R. Cohn, Über das Verhalten einiger Pyridin- und Naphtalinderivate im tierischen Stoffwechsel. Zeitschr. f. phys. Chemie. 18 (1894). S. 118.

3) J. D onath, Beiträge zu den physiolog. Wirkungen und den chem. Reaktionen des Chinolins. Ber. der Dtsch. chem. Ges. 14 (1881). S. 1769.

4) L. Brieger, Antipyretische Wirkung des Chinol. tartar. Zeitschr. f. klin. Med. 4 (1882). S. 296. 
des Akridins im Tierkörper prüfen, um die an diesem leichter zu bearbeitenden Material gewonnenen Erfahrungen später auf das Chinolin zu übertragen.

Das Chinolin ist entstanden zu denken aus der Vereinigung eines Benzolkernes mit dem Pyridin. Die weitere Addition eines Benzolkernes an das Chinolin fuhrt zum Akridin. Folgende Formelbilder veranschaulichen diese Beziehungen:

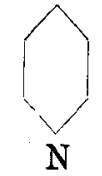

Pyridin

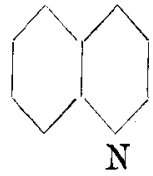

Chinolin

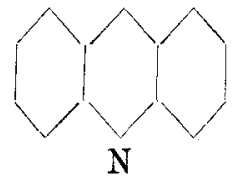

Akridin.

Leich ter mußte es von vornherein erscheinen, die Umwandlungsprodukte des Akridins, als die des Chinolins, in den Ausscheidungen des Körpers aufzufinden, da die Lösungen des ersteren und seiner Derivate hervorragende Fluoreszenz besitzen. Diese konnte wohl als Wegweiser bei der Isolierung der Stoffwechselprodukte dienen, eine Voraussetzung, welche sich in der Tat bestätigt fand.

Das Pyridin wird, wie erwähnt, als Methylpyridiniumhydroxyd (I) vom Organismus ausgeschieden. Verhält sich das Akridin wie das Pyridin, so mußte es als Methylakridiniumhydroxyd (II) in den Harn ubergehen. Dieser Körper ist bekannt; doch ist er, wie die meisten ähnlichen Hydroxyde, wenig beständig und oxydiert sich an der Luft rasch zu einem N-Metlyylakridon (III) ${ }^{1}$ ).

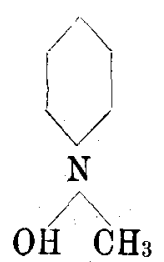

I.

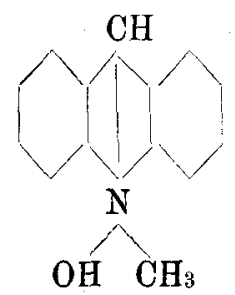

II.

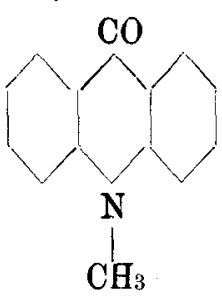

III.

Diese beständige, leicht und schön krystallisierende Substanz war als Stoffwechselprodukt des Akridins zu erwarten, wenn es sich dem Pyridin analog verhielt; Ein derartiges Methylprodukt konnte jedoch aus dem Harn nicht isoliert werden.

Es war weiterhin an eine Oxydation des Akridins im Organismus zu denken.

Wie bereits die Entdecker des Akridins, Graebe und Caro,

1) H. Decker, Über die Einwirkung von Alkalien auf Jodalkylate der Chinolin- und Akridinreihe. Journ. f. prakt. Chem. N. F. 45 (1892). S. 193. 
feststellten, ist dasselbe gegen Oxydationsmittel sehr beständig. Es verträgt längeres Kochen mit Chromsäure ohne Veränderung. Hingegen wird einer seiner Benzolkerne beim Erhitzen mit Kaliumpermanganat in alkalischer Lösung anfgespalten unter Bildung von Akridinsäure (IV) (Chinolin $\alpha, \beta$ Dicarbonsäure), die ibrerseits wieder leieht in eine Chinolinmonocarbonsäure abergeht '). Weder Chinolin- noch Pyridinearbonsäuren konnten indes aus dem Akridinharn dargestellt werden.

Durch Oxydation mit Natriumbichromat und Eisessig ${ }^{2}$ ) oder vorteilhafter mit Chlorkalk und Kobaltnitrat ${ }^{3}$ ) läßt sich das Akridin in einen sehr beständigen Körper, das Akridon (V), uberfuhren, welehes isomer ist mit dem durch Erhitzen von Anilinoakridin mit Salzsäure erhaltenen Oxyakridin (VI) ${ }^{4}$ ).
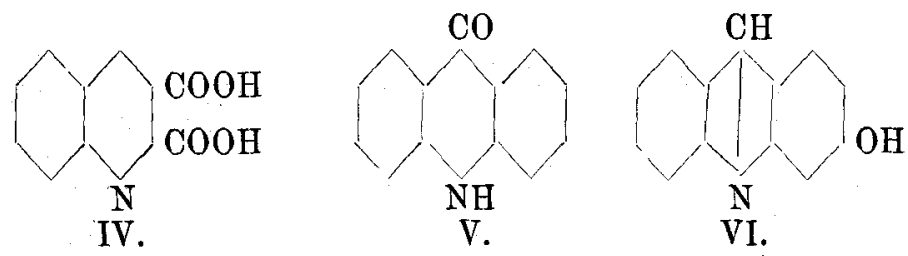

Ein solches Oxyakridin war nach Analogie mit dem Verhalten von Benzol, Naphtalin u. a. als Stoffwechselprodukt zu erwarten.

Parallel mit der Menge des verftitterten Akridins stieg die Menge der gepaarten Schwefelsäure im Harn, weleher Umstand sehr flir die Umwandlung des Akridins in ein Oxyakridin sprach. Die aus dem Harn isolierte Substanz erwies sich aber bei der Verbrennung als ein höher oxydiertes Produkt des Akridins und mußte entweder ein Dioxyakridin (VII) oder ein Oxyakridon (VIII) sein.

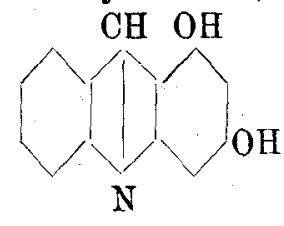

VII.

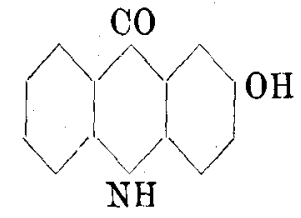

VIII.

Oxyakridone sind bis jetzt, meines Wissens, chemisch nicht dargestellt, hingegen ist ein Dioxyakridin bekannt. Das Stoffwechselprodukt des Akridins entspricht in seinem Verhalten nicht dem syn-

1) C. Graebe u. H. Caro, Über Akridin. Ber. d. Dtsch. chem. Ges. 13 (1880). S. 99 .

2) C. Graebe u. K. Lagodzinski, Über Akridon. Liebigs Ann. d. Chem. 276 (1893). S. 51.

3) A. Pictet u. E. Patry, Über Phenanthridon. Ber. d. Dtsch. chem. Ges. 26 (1893). S. 1965.

4) E. Besthorn u. W, Curtmann, Uber Anilido- und Oxyakridine. Ibidem. 94 (1891). S. 2043. 
thetisch durch Kondensation von o-Aminobenzaldehyd mit Phlorogluein dargestellten Dioxyakridin ${ }^{1}$ ), sondern hat neben den durch die Hydroxylgruppe bedingten Eigenschaften noch den Charakter eines Akridons. Die Substanz besitzt die typische Fluoreszenz der Akridone und bildet, wie diese, und im Gegensatz zu den Oxyakridinen, mit verdünter Salzsäure keine Salze. Das dargestellte Benzoylprodukt besitzt nur eine Benzoylgruppe, das Stoffwechselprodukt also nur eine Hydroxylgruppe und ist demnach ein Oxyakridon.

Über die Stellung dieser Hydroxylgruppe kann nichts Bestimmtes ausgesagt werden. Analogien zufolge (Nöltingsche Regel) könnte man für die Hydroxylgruppe Parastellung zum Stickstoff erwarten. Das erhaltene Oxyakridon wäre dann ein $5 \mathrm{Keto-3} O \times \mathrm{y}-5,10$ Dihydroakridin. ${ }^{2}$ )

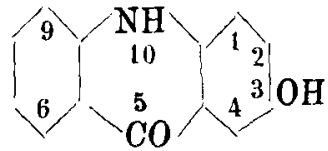

Über dasVerhalten des Akridins im Tierkörper läßt sich zusammenfassend folgendes aussagen:

Das Vorhandensein der Benzolkerne in demselben bedingt, daß es sich dem Benzol analog verhalt and sich unter Sauerstoffanfnahe und Wasseraustritt mit Schwefelsäure paart ${ }^{3}$ ). (Phenolsauerstoff.) Es ist aber nicht ausgeschlossen, dab essich als Pyridinderivat zugleich dem Pyridin analog verhalt; daß sich im Organismus zuerst Methylakridiniumbydroxyd (II), daraus $\mathrm{N}$ Methylakridon (III) bildet; und daß aus letzterem durch Entmethylierung Akridon(V) entsteht. (Ketonsauerstoff.)

Experimentelles.

Das meist ziemlich stark verunreinigte Akridin des Handels wurde in verdtinnter Salzsäure heiß gelöst, die Lösung filtriert und das Filtrat reichlich mit konq. Salzsäure versetzt. Beim Erkalten sehied sich salzsaures Akridin aus der goldgelben Lösung in langen

1) J. Eli asberg u. E. F ri ed lănd e r, Über einige Kondensationen des o-Amidobenzaldehyds. Ber. d. Dtsch. chem. Ges. 25 (1892). S. 1759.

2) Herr Dr. F. Ullmann, Genf, welchem ich eine Probe meines Oxyakridons gesandt hatte, teilte mir während der Korrektur mit, daß er denselben Körper synthetisch dargestellt habe, und daß dieser die von mir angenommene Konstitution ( $p$-Stellung des $\mathrm{OH}$ zum $N$ ) besitze.

3) Über die dieser Paarung zugrunde liegende theoretische Vorstellung vergl. O. Schmiedeberg, Ober Oxydationen und Synthesen im Tierkörper. Dieses Archiv, 14 (1881). S. 303. 
Nadeln aus. Es wurde aus salzsäurehaltigem Alkohol umkristallisiert, das so gewonnene Präparat im Verhältnis von 1:50 in Wasser gelöst und Kaninchen durch die Schlundsonde in den Magen injiziert. Die Tiere bekamen täglich, je nach der Größe, 0,6 bis $1 \mathrm{~g}$ salzsaures Akridin, welches sie längere Zeit hindurch ganz gut vertrugen ${ }^{1)}$. Fraßen die Tiere schlecht, so wurde einige Tage mit den Injektionen ausgesetzt. Das Futter der Versuchstiere bestand in Brot und Mohr- oder Zuckerrüben. Die tägliche Harnmenge betrug im Durchschnitt $300 \mathrm{ccm}$. Der bei diesem Futter normalerweise helle, trübe Urin wurde bei der Akridinfütterung klar und dunkel. Er zeigte, namentlich bei starkem Verdünnen mit Wasser, schön hellblaue Fluoreszenz und konservierte sich lange, ohne zu faulen. Die Reaktion war immer schwach alkaliseh. Zucker und Eiweiß konnten nie nachgewiesen werden.

Mit konz. Salzsäure gekocht reduzierte der Harn sehr schwach Fehlingsche Lösung. Ein geringer Teil des Stoffwechselproduktes wird demnach wohl als gepaarte Glykuronsäure ausgeschieden. Die Hauptmenge erscheint aber an Schwefelsäure gebunden. Die Abhängigkeit der ausgeschiedenen gepaarten Schwefelsäure von der Menge des verfutterten Akridins wird durch die Zahlen in folgender Tabelle veranschaulicht.

Dieselben geben das Verhältnis der Gesamtschwefelsäuremenge A im Urin zur Menge der gepaarten Schwefelsäure B an. Das Futter des $2370 \mathrm{~g}$ wiegenden männlichen Kaninchens bestand während dieser Versuchsperiode aus $200 \mathrm{~g}$ Zuckerruben und $60 \mathrm{~g}$ Brot.

\begin{tabular}{|c|c|c|}
\hline $\begin{array}{c}\text { Datum } \\
\text { Juli } 1903\end{array}$ & $\begin{array}{c}\text { Verbältnis } \\
\mathrm{A} / \mathrm{B}\end{array}$ & Bemerkungen \\
\hline $\begin{array}{l}15 . \\
16 . \\
17 . \\
18 . \\
19 . \\
20 . \\
21 . \\
22 . \\
23 .\end{array}$ & $\begin{array}{r}17,85 \\
21,30 \\
18,33 \\
3,33 \\
3,26 \\
3,78 \\
17,81 \\
3,30 \\
0,87\end{array}$ & 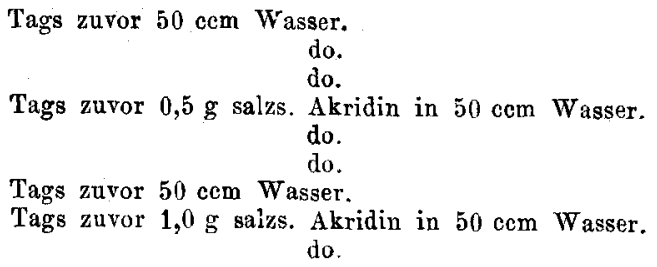 \\
\hline
\end{tabular}

Aus der Zunahme der gepaarten Schwefelsäure läßt sich zugleich berechnen, wieviel von dem verfütterten Akridin in dieser Bindung im Harn auftritt.

Nach Verfutterung von 1,0 g salzsaurem Akridin werden an 0xyakridon gebundene Schwefelsäure ausgeschieden: $0,0975 \mathrm{~g} . \quad 1,0 \mathrm{~g}$

1) Die letale Dosis beträgt $1-1,5 \mathrm{~g}$ pro kg Kaninchen. 
salzsaures Akridin (Mol.-Gew. 215,5) entsprichtnahezu derselben Menge, d.h. 0,98g Oxyakridon (Mol.-Gew. 211). Ein Molekül Oxyakridon verbindet sich mit einem Molekül Schwefelsäure (Mol.-Gew.98). 0,98 g Oxyakridon brauchen zur Bindung $0,45 \mathrm{~g}$ Schwefeläure. Der ausgeschiedenen Menge von $0,0975 \mathrm{~g}$ Sch wefelsäure entsprechen also $0,21 \mathrm{~g}$ Oxyakridon.

Dies wäre die theoretische Menge Oxyakridon, welche aus dem Harn aus $1,0 \mathrm{~g}$ verfüttertem salzsauren Akridin za erhalten sein mulfte, d. b. etwa ein Fünftel des verfutterten salzsauren Akridins findet sich im Harn als Oxyakridon. In Wirklichkeit gelingt es aber nach der unten beschriebenen Methode niemals, diese 20 Proz. des Akridinchlorhydrats in Form von Oxyakridon wiederzugewinnen. Aus $5 \mathrm{~g}$ injiziertem salzsaurem Akridin konnte ich etwa 0,2 $\mathrm{g}$ des Oxydationsprodukts erhalten, was einer Ausbeute von nur 4 Proz. entspricht.

Es wurde nach der von $B$ a $\operatorname{man}^{2}{ }^{1}$ ) angegebenen Methode versucht, die $\mathrm{Oxyakridonschwe} \mathrm{felsåure} \mathrm{C}_{6} \mathrm{H}_{4}<\underset{\mathrm{NH}}{\mathrm{CO}}>\mathrm{C}_{6} \mathrm{H}_{3} .0$. $\mathrm{SO}_{2}$. $\mathrm{OH}$ als Kalisalz zu isolieren, was jedoch nicht gelang.

Oxyakridon, $\mathrm{C}_{6} \mathrm{H}_{4}<\mathrm{NH}>\mathrm{C}_{6} \mathrm{H}_{3} . \mathrm{OH}$.

Der Harn wurde unter Zusatz von konzentrierter Salzsäure auf ein Drittel seines Volumens eingedampft und mit alkoholhaltigem Ather ausgeschtittelt. Der Äther nimmt das Oxyakridon mit gelber Farbe auf und zeigt prachtvoll hellblaue Fluoreszenz. Nachdem der Äther gewaschen war, wurde er abdestilliert und der im Kolben befindliche Krystallbrei mit wenig heißem Alkohol in ein Becherglas gespult. Die ausgeschiedenen Krystalle wurden auf ein Filter gebracht und mit Alkohol kalt gewaschen. Das aus heißem Alkohol wiederholt umkrystallisierte Präparat wurde bei gewöhnlicher Temperatur im Vakuumexsiccator uber Schwefelsäure getrocknet, und lieferte folgende analytische Daten:
1. $0,1457 \mathrm{~g}$ Substanz gaben:
2. $0,1190 \mathrm{~g}$
$0,3956 \mathrm{~g}$
$\mathrm{CO}_{2}, 0,0600 \mathrm{~g} \mathrm{H}_{2} \mathrm{O}$.
3. $0,1440 \mathrm{~g}$ $0,3230 \mathrm{~g} \mathrm{CO}_{2}, 0,0491 \mathrm{~g} \mathrm{H}_{2} \mathrm{O}$. $\mathrm{C}_{13} \mathrm{H}_{9} \mathrm{NO}_{2}$. $8,75 \mathrm{cem}$. N. $(742,5 \mathrm{~mm}, 18 \%$. Ber. C: 73,88 .
$\mathrm{H}: 4,34$. $\mathrm{N}: 6,65$. Gef. C: 74,05. 73,95. H: 4,57, 4,58. N: 6,88 .

Das Oxyakridon krystallisiert aus Alkohol in prismatischen Nadeln, welche oft blischelförmig vereint sind. Die Krystalle besitzen gelbe Farbe und braungrünen Oberflächenglanz. Der Schmelzpunkt liegt bei $327-330^{\circ}$ (unkorr.). Die Substanz löst sich sehr wenig in

1) E. Baumann, Über die Ätherschwefelsäuren der Phenole. Zeitschr. f. phys. Chem. 2 (1879). S. 335 . 
heißem Wasser und in verdünnter Salzsäure, mehr in heißer konzentrierter Salzsäure, aus welcher sie beim Erkalten krystallinisch ausfällt. Die Substanz löst sich leicht in verdünnten Alkalien, auch in Natriumkarbonat, und wird aus diesen Lösungen durch Säuren wieder ausgefällt. Kalter Äthylalkobol löst ziemlich wenig, heißer Alkohol nicht reichlich. Ebenso verhält sich Amylalkohol. In diesen beiden Alkoholen ist die Fluoreszenz rein hellblau. Die Substanz ist nahezu unlöslich in Benzol und Chloroform; sehr wenig löslich in Äther und Essigester, besser in Aceton. Die Fluoreszenz dieser Lösungen ist blaßviolett. Eisessig löst geringe Mengen "ohne Fluoreszenz. Schön grüne Fluoreszenz besitzt die Lösung in konzentrierter Schwefelsäure, und diese bleibt auch beim Verdünnen der Lösung mit Wasser bestehen. Die Farbe aller Lösungen ist rein gelb, nur die Lösungen in Alkalien besitzen gelbbraune Farbe.

Benzoyloxyakridon, $\mathrm{C}_{6} \mathrm{H}_{4}<\mathrm{NH}_{\mathrm{NH}}^{\mathrm{CO}}>\mathrm{C}_{6} \mathrm{H}_{3} .0 . \mathrm{CO} . \mathrm{C}_{6} \mathrm{H}_{5}$.

Beim Schtutteln der Lösung des Oxyakridons in verdinnter Natronlauge mit Benzoylchlorid bildete sich sofort ein graugelber Niederschlag; derselbe wurde mit Wasser und kaltem Alkohol gewaschen und aus heißem Aceton und Alkohol verschiedene Male umkrystallisiert. Die Verbrennung der im Vakuumexsiccator uber Schwefelsäure getrockneten Substanz ergab:

$0,1100 \mathrm{~g}$ Substanz: 0,3055 $\mathrm{CO}_{2}, 0,0470 \mathrm{H}_{2} \mathrm{O}$.

$\mathrm{C}_{20} \mathrm{H}_{13} \mathrm{NO}_{3}$. Ber. C: 76,20 . $\mathrm{H}: 4,1$

Gef. $\mathrm{C}: \mathbf{7 5 , 7 4}$. H: 4,74.

Die Werte für ein Dibenzoyldioxyakridin wären:

$\mathrm{C}_{27} \mathrm{H}_{17} \mathrm{NO}_{4}$. Ber. C: 77,3. $\mathrm{H}: 4,1$.

Das Benzoylprodukt krystallisiert aus der Alkohollösung in warzigen Aggregaten, welche makroskopisch aus derben Prismen zu bestehen scheinen, sich aber unter dem Mikroskop in ein Haufenwerk feiner verfilzter Nadeln auflösen. Die Krystalle sind kaum gelblich gefärbt. Der Schmelzpunkt liegt bei $265^{\circ}$ (unkorr.). Die Substanz ist unlöslich in Wasser und verdünnter Salzsäure, mit dunkelgelber Farbe löslich in heißer verdünnter Natronlauge. Sie ist mit blaßgelber Farbe ziemlich gut löslich in heißem Alkohol und Aceton; die Lösungen besitzen blaßviolette Fluoreszenz. In Äther und Benzol ist das Produkt unlöslich, kaum löslich in Chloroform, etwas reichlicher in Eisessig. Auch diese Lösungen besitzen violette Fluoreszenz. Konzentrierter Sohwefelsäure gegenüber verhält sich das Benzoylprodukt, wie das Oxyakridon selbst. 